\title{
Midterm Functional Recovery of Total Knee Arthroplasty Patients Compared Between the ATTUNE Knee System and the Press Fit Condylar (PFC) SIGMA Knee System
}

\section{Ekasame Vanitcharoenkul}

Mahidol University Faculty of Medicine Siriraj Hospital

Aasis Unnanuntana ( $\square$ uaasis@gmail.com )

Mahidol University Faculty of Medicine Siriraj Hospital https://orcid.org/0000-0002-5742-298X

\section{Research article}

Keywords: functional recovery, total knee arthroplasty, TKA, ATTUNE, Press Fit Condylar, PFC

Posted Date: April 9th, 2021

DOI: https://doi.org/10.21203/rs.3.rs-403415/v1

License: (c) (1) This work is licensed under a Creative Commons Attribution 4.0 International License. Read Full License

Version of Record: A version of this preprint was published at BMC Musculoskeletal Disorders on July 13th, 2021. See the published version at https://doi.org/10.1186/s12891-021-04464-6. 


\section{Abstract \\ Background}

The ATTUNE Knee System is a new prosthetic design that has theoretical advantages over the Press Fit Condylar (PFC) Sigma Knee System specific to improved knee kinematics and patellofemoral joint tracking. This study aimed to evaluate functional outcomes compared between the ATTUNE and PFC Sigma designs at a minimum follow-up of 5 years.

\section{Methods}

We retrospectively reviewed data from total knee arthroplasty patients who received either the ATTUNE or PFC Sigma system during November 2013 to February 2015 at Siriraj Hospital (Bangkok, Thailand). Functional outcomes were evaluated using timed up and go test, 2-minute walk test, modified knee score, numerical rating scale-pain, range of motion (ROM), and rate of anterior knee pain and crepitation at preoperation, 3-months, 1-year, and 5-years, postoperatively.

\section{Results}

Of 113 patients, 59 and 54 received the PFC Sigma and ATTUNE systems, respectively. At a minimum of 5-years follow-up, all functional outcomes improved significantly. Although the mean ROM at 5-years postoperatively was significantly higher in ATTUNE than in PFC Sigma, the difference was small $\left(116^{\circ} \mathrm{vs}\right.$. $110^{\circ}$, respectively; $p=0.041$ ). There were no significant differences in any of the other outcome measurements, including anterior knee pain, clunking, and crepitation, between groups at any study time point.

\section{Conclusions}

Our results revealed no major differences in functional outcomes between the PFC Sigma and ATTUNE TKA designs at an intermediate-term follow-up of at least 5 years. Longer-term follow-up study is needed to evaluate the benefits of the ATTUNE design relative to polyethylene wear and the rate of aseptic loosening.

\section{Background}

Total knee arthroplasty (TKA) is a highly successful orthopedic surgical procedure that delivers pain relief, functional restoration, and enhanced mobility [1-3]. Having acknowledged the commonly realized benefits of TKA, several studies have reported unsatisfactory outcomes after TKA, including unsatisfactory postoperative functional scores [4], patellofemoral complication [5], and residual pain [6]. Previous studies reported rates of dissatisfaction that ranged from $10-20 \%[4-6]$. 
To improve patient satisfaction, several different knee prosthesis designs have been developed. The Press Fit Condylar (PFC) Sigma Knee System (DePuy Synthes, Warsaw, IN) (hereafter PFC Sigma), which

is one of the most commonly used TKA designs, was initially introduced with a dome-shaped patella and a single radius trochlear groove design in $1996[7,8]$. This prosthesis is available with a range of options, including fixed-bearing (FB), rotating-platform (RP), cruciate-retaining (CR), and posterior-stabilized (PS) components. This TKA system has shown excellent long-term survivorship $[9,10]$.

The ATTUNE Knee System (DePuy Synthes) (hereafter ATTUNE) is a newer-generation TKA that was introduced in 2011 with modifications to the following femoral and tibial components: multi-radius transition from distal to posterior condyle of the femoral component, reduced femoral component profile, improved locking mechanism of the tibial base, improved anatomic trochlear groove, and medialized patellar shape. It is thought that these design modifications to more closely replicate the anatomy of the human knee could reduce pain and crepitation, leading to better functional outcome and improved patient satisfaction. However, the results of comparisons between the ATTUNE knee system and other TKA prostheses remain limited. Patient-reported outcomes of the ATTUNE TKA showed superior results over those of the PFC Sigma at 6 months after surgery; however, the number of recruited patients was small, and the follow-up period was short [11]. Another study, which was conducted by the pioneer designer of the ATTUNE TKA, concluded that the ATTUNE TKA could reduce the incidence of anterior knee pain and crepitation compared with the PFC Sigma prosthesis with no differences in Knee Society Score (KSS) or patient satisfaction [7]. In addition to the aforementioned paucity of comparative ATTUNE TKA data, no studies have compared performance-based outcomes, which are believed to truly reflect the actual function, balance, and walking ability of patients, between the ATTUNE TKA design and the PFC Sigma design.

Accordingly, the objective of this study was to compare functional outcomes, specifically performancebased measures, between the ATTUNE and PFC Sigma TKA knee systems at a minimum follow-up of 5 years.

\section{Methods}

The protocol for this study was reviewed and approved by the Siriraj Institutional Review Board (SIRB) of the Faculty of Medicine Siriraj Hospital, Mahidol University, Bangkok, Thailand (COA no. 726/2016). A retrospective review of data from a cohort group of patients who were diagnosed with end-staged osteoarthritis (OA) of the knee and who underwent TKA at our center during November 2013 to February 2015 was performed. Patients aged 40-100 years who received TKA with either the ATTUNE or PFC Sigma TKA system were eligible for inclusion. Patients who had contralateral knee pain; a history of high tibial osteotomy or who underwent a complex surgical procedure that required a metal augment or stem during the operation; and/or, who had complications or a severe medical condition that affected the standard rehabilitation protocol were excluded.

\section{Operative procedure and postoperative care}


All surgeries were performed by a single surgeon (AU) using a medial parapatellar approach. A tourniquet was applied in all cases. Standard surgical techniques were performed in all cases, including midline skin incision, subluxation of the patella, intramedullary distal femoral resection, and extramedullary proximal tibial resection. Appropriate size and rotation of the femoral component was determined using anterior referencing and the gap balancing technique, respectively. The patella was selectively resurfaced, as needed, according to the intraoperative findings. If cartilage on the patella was generally preserved with adequate patellofemoral congruency, and there was no history of crystalline or inflammatory synovitis, the patella was not resurfaced [12]. All components were cemented posterior-stabilized and fixed-bearing design. Antibiotic was given for approximately 24 hours after surgery. Intravenous fluid and Foley catheter were typically removed on the second postoperative day. Following TKA, all patients received the same postoperative rehabilitation protocol, including early mobilization and full weight-bearing with a walker as soon as possible. Postoperative pain was controlled with a combination of oral analgesics, including acetaminophen, non-steroidal anti-inflammatory drugs (NSAIDs), muscle relaxant, and oral opioids, as needed. Intravenous morphine was administered when the patient's pain score was $\geq 5$. The goal was to maintain the patient's pain score at no higher than 2-3 out of 10 as measured by numerical rating scale-pain (NRS-pain). Deep vein thrombosis prophylaxis was performed using mechanical prophylaxis, and medical prophylaxis if indicated. Patients were discharged home and instructed to perform simple knee exercises using a walker a few times a day for approximately 6 weeks.

\section{Assessment of outcomes}

Demographic data and clinical information were collected, including age, gender, body mass index (BMI), side of the operation, Charlson Comorbidity Index $(\mathrm{CCl})$, use of walking assistance device, preoperative alignment, range of motion (ROM), and modified knee score. Alignment of the knee was determined using hip knee axis (HKA), and alignment was classified as varus alignment if the HKA was $<178^{\circ}$, and valgus alignment if the HKA was $>182^{\circ}$ [13]. Our primary outcome of interest was the timed up and go (TUG) test. Secondary outcomes included 2-minute walk test (2MWT), NRS-pain, ROM, modified knee score, postoperative complications, and the percentage of patients who reported crepitation, patellar clunk, or anterior knee pain. All outcome measures were collected during the preoperative period as baseline data, and at 3-months, 1-year, and 5-years postoperatively.

\section{Timed up and go (TUG) test}

The TUG test is a reliable and validated outcome that has been used to evaluate the physical performance of patients with TKA [14]. Participants were asked to stand up from a seated position on a standard chair, walk for 3 meters, turn around, walk back to the chair, and then sit back down. They were allowed to use their arms when rising from and returning to a seated position. The time used to perform this task was measured in seconds $[14,15]$. Patients were asked to perform this test 2 times, and the average time was used for analysis.

\section{Two-minute walk test (2MWT)}


Patients were instructed to walk for 2 minutes at their normal pace up and down a designated corridor, turning around at each end of the corridor without stopping [16]. They were permitted to use a walking aid if they wanted to do so. The results were recorded as total distance walked in meters. Similar to the TUG test, the 2MWT has been validated to evaluate function after TKA [13].

\section{Modified knee score}

This scoring system was modified from the original Knee Society Clinical Rating System that was published by Insall in 1993 [17]. The modified knee scoring system is based on 3 aspects: pain, ROM, and stability. The summation of the individual scores from each aspect is then reduced by the total of the scores given for extension lag, flexion contracture, malalignment, and pain at rest. The resulting score is the patient's overall modified knee score. The highest possible score is 100 points, with higher scores indicating improved function of the knee, and lower scores indicating worsened knee function.

\section{Numerical rating scale-pain (NRS-pain)}

The level of postoperative knee pain experienced by patients while performing daily activities was assessed using the NRS-pain score. NRS-pain is a subjective measurement of pain on an eleven-point numerical scale that ranges from 0 (no pain) to 10 (the worst imaginable pain). NRS-pain is efficient for use in clinical practice, and showed good test-retest reliability in different populations [18].

\section{Crepitation, patellar clunk, and anterior knee pain}

Patellofemoral crepitus or crepitation is defined as painless hearable noises and/or palpable vibrations from the knee of a TKA patient. Patellar clunk was first described by Hozack, et al. [19], and is defined as a painful catching, grinding, or jumping of the patella when the knee moves from a flexed to an extended position after total knee replacement [20]. Anterior knee pain is a subjective symptom of pain around the anterior part of the knee [21].

\section{Statistical analysis}

Based on the results of a previous investigation [13] that reported the minimal clinically important difference (MCID) of TUG at 1 year to be 9.5, a power analysis conducted a priori determined that a minimum of 46 subjects per group was required to establish a minimum effect size of this MCID value with a 2-sided alpha level of 0.05 and $90 \%$ power. Descriptive statistics are presented as mean and standard deviation for continuous variables, and as frequency and percentage for categorical variables. Student's unpaired $t$-test was used to compare quantitative variables between two groups. Categorical data, such as the rate of patients who had anterior knee pain and crepitation, were analyzed using either Fisher's exact test or chi-square test. Changes in each outcome measurement from baseline to 3 months, 1 year, and 5 years after TKA were assessed using one-way repeated mesures analysis of variance (oneway repeated measures ANOVA). Analysis of covariance (ANCOVA) was used to adjust significant demographic data and baseline performance-based measurements to provide an unbiased difference in performance-based outcome at 5-years post-TKA. Other outcome measures were analyzed using Student's unpaired $t$-test to compare differences between the two prosthesis systems at each follow-up 
time point. Statistical analyses were performed using SPSS Statistics for Windows, version 18.0 software (SPSS, Inc., Chicago, IL, USA). Statistical significance was defined as a $p$-value less than 0.05 .

\section{Results}

From a total of 155 patients, 42 were excluded due to the following conditions: contralateral knee pain (n $=25)$, received TKA using other prosthesis designs $(n=14)$, active spinal disease $(n=1)$, underwent complex primary TKA $(n=1)$, and had periprosthetic fracture at 2 months after the index surgery $(n=1)$. The remaining 113 patients were enrolled in this study. Of those, 59 and 54 patients underwent TKA using the PFC Sigma and ATTUNE knee systems, respectively. The mean follow-up duration was 75.3 months (range: 60.0 to 92.0 ). Of the 9 patients who underwent patellar resurfacing, 6 patients $(10.2 \%)$ and 3 patients $(5.6 \%)$ were in the PFC Sigma group and the ATTUNE group, respectively $(p=0.366)$.

Patient demographic and clinical characteristics are shown in Table 1. The average age of patients was 71.1 years, and most of them (89.4\%) were female. The mean age of patients in the PFC Sigma and ATTUNE TKA groups was 70.0 and 73.4 years, respectively $(p=0.023)$. The majority of patients had CCI in the range of $0-3$ in the PFC Sigma group (59.3\%), and higher than 3 in the ATTUNE group (55.6\%). There were no significant differences in gender or $\mathrm{CCl}$ distribution between the two groups. Most patients had varus knee alignment before TKA (81.4\%). The mean HKA among all patients was 169.9 degrees. The mean preoperative ROM was 102.5 and 100.5 degrees in the PFC Sigma and ATTUNE groups, respectively. There were no significant preoperative differences between groups relative to gait aid use, knee alignment, ROM, or modified knee score. 
Table 1

Patient demographic and clinical characteristics

\begin{tabular}{|c|c|c|c|}
\hline Characteristics & $\begin{array}{l}\text { PFC Sigma } \\
(n=59)\end{array}$ & $\begin{array}{l}\text { ATTUNE } \\
(n=54)\end{array}$ & $p$-value \\
\hline Age (years), mean \pm SD & $70.0 \pm 8.2$ & $73.4 \pm 7.2$ & 0.023 \\
\hline Female gender, $\mathrm{n}(\%)$ & $51(86.4 \%)$ & $50(92.6 \%)$ & 0.367 \\
\hline Body mass index $\left(\mathrm{kg} / \mathrm{m}^{2}\right)$, mean $\pm \mathrm{SD}$ & $26.5 \pm 4.9$ & $26.2 \pm 3.7$ & 0.679 \\
\hline Right side, n (\%) & $30(50.8 \%)$ & $23(42.6 \%)$ & 0.452 \\
\hline Charlson Comorbidity Index, n (\%) & $35(59.3 \%)$ & $24(44.4 \%)$ & 0.114 \\
\hline $0-3$ & $24(40.7 \%)$ & $30(55.6 \%)$ & \\
\hline \multicolumn{4}{|l|}{$>3$} \\
\hline Gait aid, n (\%) & $33(60.0 \%)$ & $20(37.0 \%)$ & 0.146 \\
\hline None & $13(22.0 \%)$ & $17(31.5 \%)$ & \\
\hline Cane & $12(20.3 \%)$ & $17(31.5 \%)$ & \\
\hline Walker & $1(1.7 \%)$ & $0(0.0 \%)$ & \\
\hline \multicolumn{4}{|l|}{ Wheel chair } \\
\hline Hip knee axis (degrees), mean \pm SD & $170.0 \pm 10.9$ & $169.8 \pm 8.1$ & 0.912 \\
\hline Varus alignment, n (\%) & $47(79.7 \%)$ & $45(83.3 \%)$ & \\
\hline Valgus alignment, n (\%) & $7(11.9 \%)$ & $2(3.7 \%)$ & \\
\hline Preoperative range of motion (degrees), mean \pm SD & $102.5 \pm 18.2$ & $100.5 \pm 16.3$ & 0.538 \\
\hline Preoperative modified knee score, mean \pm SD & $44.9 \pm 11.4$ & $44.9 \pm 10.2$ & 0.978 \\
\hline \multicolumn{4}{|l|}{ A $p$-value $<0.05$ indicates statistical significance. } \\
\hline \multicolumn{4}{|l|}{ Varus alignment is defined as tibiofemoral angle $<178^{\circ}$} \\
\hline \multicolumn{4}{|l|}{ Valgus alignment is defined as tibiofemoral angle $>182^{\circ}$} \\
\hline Abbreviation: PFC, Press Fit Condylar; SD, standarc & viation & & \\
\hline
\end{tabular}

The mean preoperative performance-based test results in the PFC Sigma and ATTUNE groups were 21.5 and 23.9 seconds for the TUG test, and 45.8 and 45.3 meters for the 2MWT, respectively. At 1-year postTKA, the time needed to complete the TUG test was reduced to 14.3 and 15.6 seconds in the PFC Sigma and ATTUNE groups, respectively. Similarly, the distance walked within 2 minutes increased to 69.4 and 
63.8 meters in the PFC Sigma and ATTUNE groups, respectively, at the 1-year follow-up time point (Table 2). At 5-years post-TKA, both of these 2 performance-based measures declined significantly, but only in the PFC Sigma group ( $p=0.017$ and $p=0.002$ for TUG and 2MWT, respectively) (Fig. 1 ). There were no significant differences in these two performance-based measures between the PFC Sigma and ATTUNE groups at any study time point (Table 2). 
Table 2

Outcome measurements compared between the PFC Sigma and ATTUNE systems at baseline, and at the 3-month, 1-year, and 5-year postoperative follow-ups

\begin{tabular}{|c|c|c|c|}
\hline Outcome measurement & $\begin{array}{l}\text { PFC Sigma } \\
(n=59) \\
\text { Mean } \pm \text { SD }\end{array}$ & $\begin{array}{l}\text { ATTUNE } \\
(n=54) \\
\text { Mean } \pm \text { SD }\end{array}$ & $p$-value \\
\hline Timed up and go test & $21.5 \pm 10.9$ & $23.9 \pm 14.8$ & 0.425 \\
\hline Baseline & $17.3 \pm 7.2$ & $19.4 \pm 9.2$ & 0.276 \\
\hline 3 months & $14.3 \pm 5.6$ & $15.6 \pm 5.6$ & 0.322 \\
\hline $\begin{array}{l}1 \text { year } \\
5 \text { years }\end{array}$ & $16.9 \pm 9.9$ & $16.8 \pm 5.6$ & 0.949 \\
\hline Two-minute walk test & $45.8 \pm 21.5$ & $45.3 \pm 18.5$ & 0.919 \\
\hline Baseline & $60.4 \pm 20.7$ & $55.6 \pm 18.2$ & 0.293 \\
\hline 3 months & $69.4 \pm 19.0$ & $63.8 \pm 15.6$ & 0.164 \\
\hline $\begin{array}{l}1 \text { year } \\
5 \text { years }\end{array}$ & $64.1 \pm 64.2$ & $62.5 \pm 16.2$ & 0.709 \\
\hline Numerical rating scale-pain & $7.4 \pm 1.9$ & $7.7 \pm 1.6$ & 0.447 \\
\hline Baseline & $1.6 \pm 1.3$ & $1.5 \pm 0.9$ & 0.560 \\
\hline 3 months & $0.8 \pm 1.2$ & $0.6 \pm 0.7$ & 0.561 \\
\hline $\begin{array}{l}1 \text { year } \\
5 \text { years }\end{array}$ & $0.3 \pm 0.6$ & $0.4 \pm 0.8$ & 0.734 \\
\hline Range of motion (degrees) & $102.0 \pm 21.6$ & $98.9 \pm 13.6$ & 0.522 \\
\hline Baseline & $105.7 \pm 11.2$ & $105.3 \pm 7.6$ & 0.855 \\
\hline 3 months & $106.5 \pm 10.3$ & $109.6 \pm 7.9$ & 0.203 \\
\hline 1 year & $110.0 \pm 10.8$ & $115.8 \pm 9.9$ & 0.041 \\
\hline 5 years & & & \\
\hline
\end{tabular}

A $p$-value $<0.05$ indicates statistical significance Abbreviation: PFC, Press Fit Condylar; SD, standard deviation 


\begin{tabular}{|llll|}
\hline Outcome measurement & $\begin{array}{l}\text { PFC Sigma } \\
(\mathbf{n = 5 9 )} \\
\text { Mean } \pm \text { SD }\end{array}$ & $\begin{array}{l}\text { ATTUNE } \\
(\mathbf{n}=\mathbf{5 4})\end{array}$ & Mean \pm -vDalue \\
\hline Modified knee score & $44.9 \pm 11.3$ & $45.2 \pm 10.5$ & 0.884 \\
Baseline & $87.2 \pm 6.5$ & $87.5 \pm 4.1$ & 0.761 \\
3 months & $90.9 \pm 7.9$ & $91.4 \pm 6.1$ & 0.696 \\
1 year & $93.8 \pm 6.4$ & $95.0 \pm 4.2$ & 0.276 \\
5 years & & & \\
\hline A p-value < 0.05 indicates statistical significance & \\
\hline Abbreviation: PFC, Press Fit Condylar; SD, standard deviation \\
\hline
\end{tabular}

During the first year after surgery, the postoperative NRS-pain score and the modified knee score were both significantly improved with both peaking at 1-year post-TKA. Afterwards, the mean NRS-pain score in the PFC Sigma group significantly improved from 1 point at 1-year post-TKA to 0.5 point at 5-years post-TKA ( $p=0.017)$; however, there was no significant change in the NRS-pain score in the ATTUNE group ( $p=0.124)$ (Fig. 1). There was also no significant difference in the NRS-pain score or the modified knee score between these two prosthetic designs at any study time point (Table 2). Although the 1-year postoperative ROM increased from the preoperative period, the difference was statistically significant only in the ATTUNE TKA group. Thereafter, ROM continued to improve throughout the 5-year postoperative period in both study groups (Fig. 1). The mean postoperative ROM at 5-years post-TKA was significantly higher in the ATTUNE group than in the PFC Sigma group (115.8 and 110.0 degrees, respectively; $p=0.041$ ) (Table 2 ).

Postoperative anterior knee pain was observed in 1 patient, and that patient received a PFC Sigma. Crepitation was found in 2 patients in the PFC Sigma group, and in 4 patients in the ATTUNE group. There was no significant difference between groups relative to anterior knee pain $(p=1.000)$ or crepitation $(p=$ 0.423). No patients in this study developed patellar clunk syndrome. There were 6 patients who developed stiffness after TKA that required manipulation under anesthesia at 3 months after surgery (2 and 4 patients in the PFC Sigma and ATTUNE groups, respectively, $p=0.708)$. One patient in the PFC Sigma group had wound dehiscence that required debridement and resuturing. No patients in this study developed aseptic loosening or superficial/deep infection after TKA (Table 3). 
Table 3

Results of anterior knee pain, crepitation, patellar clunk, and postoperative complications

\begin{tabular}{|llll|}
\hline Outcome measurement & $\begin{array}{l}\text { PFC Sigma } \\
(\mathbf{n = 5 9 )}\end{array}$ & $\begin{array}{l}\text { ATTUNE } \\
(\mathbf{n}=\mathbf{5 4})\end{array}$ & p-value \\
\hline Anterior knee pain, $\mathrm{n}(\%)$ & $1(1.7 \%)$ & $0(0.0 \%)$ & 1.000 \\
\hline Asymptomatic crepitation, $\mathrm{n}(\%)$ & $2(3.4 \%)$ & $4(7.4 \%)$ & 0.423 \\
\hline Complication, $\mathrm{n}(\%)$ & $3(5.1 \%)$ & $4(7.4 \%)$ & 0.708 \\
- Stiff knee & $2(3.4 \%)$ & $4(7.4 \%)$ & \\
- Wound dehiscence & $1(1.7 \%)$ & $0(0.0 \%)$ & \\
\hline A $p$-value < 0.05 indicates statistical significance & \\
\hline Abbreviation: PFC, Press Fit Condylar & \\
\hline
\end{tabular}

\section{Discussion}

TKA is one of the most frequently performed procedures in orthopedic surgery due to its proven efficacy for relieving pain and restoring mobility in patients with knee osteoarthritis. The proportion of patients that will need to undergo this procedure is expected to increase in Asian countries. Although many studies reported improvement in functional outcome after TKA, a substantial proportion of patients still described some level of dissatisfaction with the outcome of TKA. As a result, newer prosthetic designs have been developed to improve functional outcomes and to reduce the risk of complications. The ATTUNE knee system is one of those more recently designed knee systems, and its key design characteristics are modifications of two predecessor models from the same company (i.e., PFC Sigma, and Low Contact Stress [LCS] Complete Knee System). The improved anatomical design of the ATTUNE system is expected to deliver superior clinical outcomes to those expected from the PFC Sigma. Moreover, the longevity of the polyethylene is also expected to be longer in the ATTUNE prosthetic knee compared to the PFC Sigma. Studies that have compared the intermediate-term results of the ATTUNE system with other knee prosthesis systems are limited.

The ATTUNE TKA system, which represents an advancement in knee replacement technology, was expected to deliver better performance than the PFC Sigma TKA system. However and in contrast, the results of our study revealed no significant differences between the ATTUNE and PFC Sigma knee systems relative to pain, modified knee score, or performance-based measures during a minimum followup of 5 years. White, et al. [22] conducted a combined retrospective and prospective cohort matched-pair study that found significantly less residual pain (using yes or no questions) in the ATTUNE group than in the PFC Sigma group at the 5-year follow-up (19.5\% vs. 36.3\%, respectively; $p=0.02)$. However, when Knee Society Score (KSS) and the Western Ontario and McMaster University Osteoarthritis Index (WOMAC) were compared between the two knee systems, no significant difference between groups was 
observed for either measure. In addition - in those who reported residual pain, the pain symptom was only mild and occasional. Therefore, this residual pain might not be clinically important. Similar to our results, they reported no significant differences in functional outcomes, including the rate of anterior knee pain, crepitation, or modified knee score, between the two groups.

Previous studies showed that the post-TKA ROM plateaued at approximately 6 to 12 months postoperatively $[23,24]$. Conversely, our study showed that the ROM continued to increase from 1-year to 5 -years postoperatively in both evaluated knee systems. This may be explained by the fact that as people get older, there is a tendency for soft tissue to become more relaxed, which can lead to increased ROM [25]. Additionally, the mean ROM in the ATTUNE group was significantly higher than that of the PFC Sigma group (115.8 vs. 110 degrees, respectively; $p=0.041)$ at 5 -years post-TKA; however, there was no significant difference between groups for TKA stability. Since ROM is multifactorial, the reason for the difference in ROM at 5 years between these two prosthetic designs is still unclear. It is possible that the ATTUNE design may provide a late improvement in ROM compared to that of the PFC Sigma. Nevertheless, the 5.8-degree gain in the ATTUNE TKA group may not be clinically important.

Although most previous studies showed no major differences between the ATTUNE and PFC Sigma systems relative to functional outcomes during a short-term follow-up [7, 26-30] (Table 4), some studies reported that the ATTUNE system had less patients who experienced crepitation and pain. Martin, et al. [29] found a lower rate of crepitation in the ATTUNE group than in the PFC Sigma group at the 2-year follow-up (0.83\% vs. 9.4\%, respectively). Ranawat, et al. [7] reported that ATTUNE patients had less incidence of anterior knee pain and crepitation than PFC Sigma patients; however, a longer-term follow-up study at 5-years postoperatively of the same population found no significant difference in the incidence of anterior knee pain between groups ( $11.7 \%$ vs. $22.1 \%$ for the ATTUNE and PFC Sigma groups, respectively; $p=0.09$ ) [22]. The authors suggested explanation for this finding was that some patients in the earlier cohort were diseased and lost to follow-up. Thus, the 5-year study might have been underpowered to detect a difference in the occurrence of anterior knee pain. Song, et al. [30] reported that the ATTUNE group had a significantly higher knee score domain in the KSS (93.1 vs. 88.8 points for ATTUNE and PFC Sigma respectively, $p<0.001$ ), and that ATTUNE yielded approximately 2.4 degrees more ROM than the PFC Sigma at the 2-year follow-up. This difference, however, was still within the 7.2 points of the minimal clinically important difference (MCID) of the KSS [31]. It is also important to point out that none of those previous studies evaluated functional outcome using performance-based testing like the TUG test and 2MWT. Since patient-reported outcome measure (PROM) and performance-based testing are interchangeable and equally important [32], a comprehensive evaluation of knee function should include a combination of PROM and performance-based tests to better assess functional recovery in patients after TKA. 
Table 4

Literature review for previous studies that compared the outcome results between the PFC Sigma and ATTUNE knee systems

\begin{tabular}{|c|c|c|c|c|c|c|}
\hline Paper & $\begin{array}{l}\text { Year of } \\
\text { publication }\end{array}$ & $\begin{array}{l}\text { Type of } \\
\text { study }\end{array}$ & Duration & $\begin{array}{l}\text { Sample } \\
\text { size }\end{array}$ & Outcomes & Findings \\
\hline $\begin{array}{l}\text { Ranawat, } \\
\text { et al. [7] }\end{array}$ & 2017 & $\begin{array}{l}\text { Prospective, } \\
\text { matched-pair }\end{array}$ & 2 years & 200 & $\begin{array}{l}\text { KSS, } \\
\text { anterior } \\
\text { knee pain, } \\
\text { crepitation, } \\
\text { satisfaction } \\
\text { rate }\end{array}$ & $\begin{array}{l}\text { ATTUNE had a } \\
\text { significantly lower } \\
\text { incidence of } \\
\text { anterior knee pain } \\
(12.5 \% \text { vs. } 25.8 \% \text {, } \\
p=0.02) \text { and } \\
\text { crepitation }(17.7 \% \\
\text { Vs. } 30.9 \%, p= \\
0.02)\end{array}$ \\
\hline $\begin{array}{l}\text { Martin, et } \\
\text { al. [29] }\end{array}$ & 2017 & Retrospective & 2 years & 1,893 & $\begin{array}{l}\text { KSS, ROM, } \\
\text { crepitation }\end{array}$ & $\begin{array}{l}\text { ATTUNE had a } \\
\text { significantly lower } \\
\text { incidence of } \\
\text { crepitation }(0.8 \% \\
\text { Vs. } 9.4 \%, p< \\
0.001)\end{array}$ \\
\hline $\begin{array}{l}\text { Song, et } \\
\text { al. [30] }\end{array}$ & 2018 & Prospective & 2 years & 600 & $\begin{array}{l}\text { KSS, ROM, } \\
\text { risk of } \\
\text { patella } \\
\text { injury }\end{array}$ & $\begin{array}{l}\text { ATTUNE had a } \\
\text { significantly } \\
\text { higher knee score } \\
\text { domain in the KSS } \\
(93.1 \text { vs. } 88.8 \\
\text { points, } p<0.001) \\
\text { and ROM (131.4 } \\
\text { vs. } 129.0 \text { degrees, } \\
p=0.008)\end{array}$ \\
\hline $\begin{array}{l}\text { Chua, et } \\
\text { al. [27] }\end{array}$ & 2019 & Prospective & 2 years & 130 & $\begin{array}{l}\text { KSS, ROM, } \\
\text { Oxford } \\
\text { knee score, } \\
\text { Short Form- } \\
36 \text {, } \\
\text { satisfaction } \\
\text { rate }\end{array}$ & $\begin{array}{l}\text { No significant } \\
\text { differences in any } \\
\text { outcome } \\
\text { measurements }\end{array}$ \\
\hline $\begin{array}{l}\text { Molloy, } \\
\text { et al. [28] }\end{array}$ & 2019 & Prospective & 2 years & 2,116 & $\begin{array}{l}\text { Patient- } \\
\text { reported } \\
\text { outcome } \\
\text { measures, } \\
\text { ROM, } \\
\text { reoperative } \\
\text { rate }\end{array}$ & $\begin{array}{l}\text { No significant } \\
\text { differences in any } \\
\text { outcome } \\
\text { measurements }\end{array}$ \\
\hline $\begin{array}{l}\text { Hauer, et } \\
\text { al. [26] }\end{array}$ & 2020 & $\begin{array}{l}\text { Randomized } \\
\text { control trial }\end{array}$ & 2 years & 158 & $\begin{array}{l}\text { KSS, ROM, } \\
\text { WOMAC, } \\
\text { VAS }\end{array}$ & $\begin{array}{l}\text { No significant } \\
\text { differences in any } \\
\text { outcome } \\
\text { measurements }\end{array}$ \\
\hline
\end{tabular}




\begin{tabular}{|c|c|c|c|c|c|c|}
\hline Paper & $\begin{array}{l}\text { Year of } \\
\text { publication }\end{array}$ & $\begin{array}{l}\text { Type of } \\
\text { study }\end{array}$ & Duration & $\begin{array}{l}\text { Sample } \\
\text { size }\end{array}$ & Outcomes & Findings \\
\hline $\begin{array}{l}\text { White, et } \\
\text { al. [22] }\end{array}$ & 2020 & $\begin{array}{l}\text { Combined } \\
\text { prospective \& } \\
\text { retrospective, } \\
\text { matched-pair }\end{array}$ & 5 years & 154 & $\begin{array}{l}\text { KSS, } \\
\text { WOMAC, } \\
\text { anterior } \\
\text { knee pain, } \\
\text { crepitation, } \\
\text { residual } \\
\text { pain, } \\
\text { satisfaction } \\
\text { rate }\end{array}$ & $\begin{array}{l}\text { ATTUNE had a } \\
\text { significantly lower } \\
\text { incidence of } \\
\text { residual pain } \\
(19.5 \% \text { vs. } 36.3 \% \text {, } \\
p=0.02)\end{array}$ \\
\hline $\begin{array}{l}\text { The } \\
\text { present } \\
\text { study }\end{array}$ & & Retrospective & 5 year & 113 & $\begin{array}{l}\text { Modified } \\
\text { knee score, } \\
\text { ROM, NRS- } \\
\text { pain, TUG, } \\
\text { 2MWT, } \\
\text { anterior } \\
\text { knee pain, } \\
\text { crepitation, } \\
\text { patellar } \\
\text { clunk }\end{array}$ & $\begin{array}{l}\text { ATTUNE had a } \\
\text { significantly } \\
\text { higher ROM at the } \\
\text { 5-year } \\
\text { postoperative } \\
\text { follow-up (115.8 } \\
\text { Vs. } 110.0 \text { degrees, } \\
p=0.041 \text { ) }\end{array}$ \\
\hline
\end{tabular}

Abbreviations: PFC, Press Fit Condylar; KSS, Knee Society Score; ROM, range of motion; WOMAC, Western Ontario and McMaster Universities Osteoarthritis Index; VAS, visual analog scale for pain; TUG, timed up and go test; 2MWT, two-minute walk test; NRS-pain, numerical rating scale-pain

The strength of this study is that it is the first to compare the performance-based outcomes of two commonly used TKA designs at a minimum follow-up of 5 years. Our study also has some mentionable limitations. First, as with all retrospective studies, our study was subject to inherent biases in patient selection. Second, the study period was considered intermediate (range: 60 to 92 months), which may not be long enough to prove the longevity of these two prostheses. Further follow-up is needed to compare the survivorship of these two knee systems in this patient population. Third, our study used modified knee score, ROM, NRS-pain and two performance-based tests as outcome measurements. However, other knee functional scores and performance-based tests are available, and we are not able to recommend which of those metrics are best for evaluating function after TKA. The scores and tests that we used in this study may not be sensitive enough to detect statistically significant differences in knee function between the ATTUNE and PFC Sigma TKA designs. It is possible that patients with one prosthetic design could have had better postoperative function if they were asked to perform above the limits of the functional tests that were used in this study.

\section{Conclusions}

The results of this study revealed no significant difference in functional outcomes, including anterior knee pain, clunking, and crepitation, between the ATTUNE and PFC Sigma knee systems at a minimum followup of 5 years after TKA even though the ATTUNE design is thought to possess theoretical advantages 
that were not included in the design of the PFC Sigma. It remains unknown whether the new polytheylene in the ATTUNE TKA will reduce wear and the rate of aseptic loosening; therefore, a longer-term follow-up study evaluating the longevity and outcomes of the ATTUNE TKA is needed.

\section{Declarations}

\section{Ethic approval and consent to participate}

The protocol for this study was reviewed and approved by the Siriraj Institutional Review Board (SIRB) of the Faculty of Medicine Siriraj Hospital, Mahidol University, Bangkok, Thailand (COA no. 726/2016). Informed consent was obtained from all individual participants included in the study.

\section{Availability of data and materials}

The data collected and analyzed in the current study are available from the corresponding author on reasonable request.

\section{Consent for publication}

Not applicable.

\section{Competing interests}

All authors declare no personal or professional conflicts of interest and no financial support from the companies that produce and/or distribute the drugs, devices, or materials described in this report.

\section{Funding}

No funding was received for conducting this study.

\section{Authors' contributions}

$\mathrm{EV}$ and $\mathrm{AU}$ contributed to conception and design of the study. EV performed the data collection and the statistical analysis. EV wrote the original draft and EV contributed to reviewing and editing the manuscript. All authors read and approved the final manuscript.

\section{Acknowledgements}

The authors gratefully acknowledge the patients that agreed to participate in this study; Ms. Wachirapan Narktang, MSc for her assistance during the data collection process; and, Mr. Suthipol Udompunturak, MSc for his assistance with statistical analysis.

\section{Author detail}


Department of Orthopedic Surgery, Faculty of Medicine Siriraj Hospital, Mahidol University, 2 Wanglang Road, Bangkoknoi, Bangkok 10700, Thailand.

\section{References}

1. Brander VA, Stulberg SD, Adams AD, Harden RN, Bruehl S, Stanos SP, et al. Predicting total knee replacement pain: a prospective, observational study. Clin Orthop Relat Res. 2003:27-36.

2. Diduch DR, Insall JN, Scott WN, Scuderi GR, Font-Rodriguez D. Total knee replacement in young, active patients. Long-term follow-up and functional outcome. J Bone Joint Surg Am. 1997;79:57582.

3. Rossi MD, Hasson S, Kohia M, Pineda E, Bryan W. Mobility and perceived function after total knee arthroplasty. J Arthroplasty. 2006;21:6-12.

4. Clement ND, Burnett R. Patient satisfaction after total knee arthroplasty is affected by their general physical well-being. Knee Surg Sports Traumatol Arthrosc. 2013;21:2638-46.

5. Toomey SD, Daccach JA, Shah JC, Himden SE, Lesko JP, Hamilton WG. Comparative Incidence of Patellofemoral Complications Between 2 Total Knee Arthroplasty Systems in a Multicenter, Prospective Clinical Study. J Arthroplasty. 2017;32:187-s92.

6. Baker PN, van der Meulen JH, Lewsey J, Gregg PJ. The role of pain and function in determining patient satisfaction after total knee replacement. Data from the National Joint Registry for England and Wales. J Bone Joint Surg Br. 2007;89:893-900.

7. Ranawat CS, White PB, West S, Ranawat AS. Clinical and Radiographic Results of Attune and PFC Sigma Knee Designs at 2-Year Follow-Up: A Prospective Matched-Pair Analysis. J Arthroplasty. 2017;32:431-6.

8. Zaki SH, Rafiq I, Kapoor A, Raut V, Gambhir AK, Porter ML. Medium-term results with the Press Fit Condylar (PFC) Sigma knee prosthesis the Wrightington experience. Acta Orthop Belg. 2007;73:55-9.

9. Hunter N, Clayton RAE, Brenkel IJ. Press fit condylar Sigma total knee arthroplasty: 7-9 years results. Eur J Orthop Surg Traumatol. 2009;19:409-13.

10. Hopley CD, Dalury DF. A systematic review of clinical outcomes and survivorship after total knee arthroplasty with a contemporary modular knee system. J Arthroplasty. 2014;29:1398-411.

11. Carey BW, Harty J. A comparison of clinical- and patient-reported outcomes of the cemented ATTUNE and PFC sigma fixed bearing cruciate sacrificing knee systems in patients who underwent total knee replacement with both prostheses in opposite knees. J Orthop Surg Res. 2018;13:54.

12. Abdel MP, Parratte S, Budhiparama NC. The patella in total knee arthroplasty: to resurface or not is the question. Curr Rev Musculoskelet Med. 2014;7:117-24.

13. Unnanuntana A, Ruangsomboon P, Keesukpunt W. Validity and Responsiveness of the Two-Minute Walk Test for Measuring Functional Recovery After Total Knee Arthroplasty. J Arthroplasty. 2018;33:1737-44. 
14. Yuksel E, Kalkan S, Cekmece S, Unver B, Karatosun V. Assessing Minimal Detectable Changes and Test-Retest Reliability of the Timed Up and Go Test and the 2-Minute Walk Test in Patients With Total Knee Arthroplasty. J Arthroplasty. 2017;32:426-30.

15. Yeung TS, Wessel J, Stratford PW, MacDermid JC. The timed up and go test for use on an inpatient orthopaedic rehabilitation ward. J Orthop Sports Phys Ther. 2008;38:410-7.

16. Connelly DM, Thomas BK, Cliffe SJ, Perry WM, Smith RE. Clinical utility of the 2-minute walk test for older adults living in long-term care. Physiother Can. 2009;61:78-87.

17. Insall JN, Dorr LD, Scott RD, Scott WN. Rationale of the Knee Society clinical rating system. Clin Orthop Relat Res. 1989:13-4.

18. Stratford PW, Spadoni G. The reliability, consistency, and clinical application of a numeric pain rating scale. Physiother Can. 2001;53:88-91.

19. Hozack WJ, Rothman RH, Booth RE Jr, Balderston RA. The patellar clunk syndrome. A complication of posterior stabilized total knee arthroplasty. Clin Orthop Relat Res. 1989:203-8.

20. Fukunaga K, Kobayashi A, Minoda Y, Iwaki H, Hashimoto Y, Takaoka K. The incidence of the patellar clunk syndrome in a recently designed mobile-bearing posteriorly stabilised total knee replacement. J Bone Joint Surg Br. 2009;91:463-8.

21. Petersen W, Rembitzki IV, Brüggemann G-P, Ellermann A, Best R, Koppenburg AG, et al. Anterior knee pain after total knee arthroplasty: a narrative review. Int Orthop. 2014;38:319-28.

22. White PB, Turcan S, Satalich JR, Ranawat AS, Ranawat CS. Clinical Outcomes of a Modern Total Knee Arthroplasty Prosthesis Compared to Its Predecessor at 5-Year Follow-Up: Matched Pair Analysis. J Arthroplasty. 2020;35:3150-5.

23. Springorum HR, Maderbacher G, Craiovan B, Lüring C, Baier C, Grifka J, et al. No difference between standard and high flexion cruciate retaining total knee arthroplasty: a prospective randomised controlled study. Knee Surg Sports Traumatol Arthrosc. 2015;23:1591-7.

24. Zhou Z, Yew KSA, Arul E, Chin P-L, Tay KJD, Lo N-N, et al. Recovery in knee range of motion reaches a plateau by 12 months after total knee arthroplasty. Knee Surg Sports Traumatol Arthrosc. 2015;23:1729-33.

25. Rudolph KS, Schmitt LC, Lewek MD. Age-related changes in strength, joint laxity, and walking patterns: are they related to knee osteoarthritis? Phys Ther. 2007;87:1422-32.

26. Hauer G, Hörlesberger N, Klim S, Bernhardt GA, Leitner L, Glehr M, et al. Mid-term results show no significant difference in postoperative clinical outcome, pain and range of motion between a wellestablished total knee arthroplasty design and its successor: a prospective, randomized, controlled trial. Knee Surg Sports Traumatol Arthrosc. 2020. http://dx.doi.org/10.1007/s00167-020-06027-z.

27. Chua JL, Goh GS, Liow MHL, Tay DK, Lo NN, Yeo SJ. Modern TKA implants are equivalent to traditional TKA implants in functional and patellofemoral joint-related outcomes. Knee Surg Sports Traumatol Arthrosc. 2019;27:1116-23.

28. Molloy IB, Keeney BJ, Sparks MB, Paddock NG, Koenig KM, Moschetti WE, et al. Short term patient outcomes after total knee arthroplasty: Does the implant matter? Knee. 2019;26:687-99. 
29. Martin JR, Jennings JM, Watters TS, Levy DL, McNabb DC, Dennis DA. Femoral Implant Design Modification Decreases the Incidence of Patellar Crepitus in Total Knee Arthroplasty. J Arthroplasty. 2017;32:1310-3.

30. Song SJ, Kang SG, Park CH, Bae DK. Comparison of Clinical Results and Risk of Patellar Injury between Attune and PFC Sigma Knee Systems. Knee Surg Relat Res. 2018;30:334-40.

31. Lizaur-Utrilla A, Gonzalez-Parreño S, Martinez-Mendez D, Miralles-Muñoz FA, Lopez-Prats FA. Minimal clinically important differences and substantial clinical benefits for Knee Society Scores. Knee Surg Sports Traumatol Arthrosc. 2020;28:1473-8.

32. Bolink SA, Grimm B, Heyligers IC. Patient-reported outcome measures versus inertial performancebased outcome measures: A prospective study in patients undergoing primary total knee arthroplasty. Knee. 2015;22:618-23.

\section{Figures}



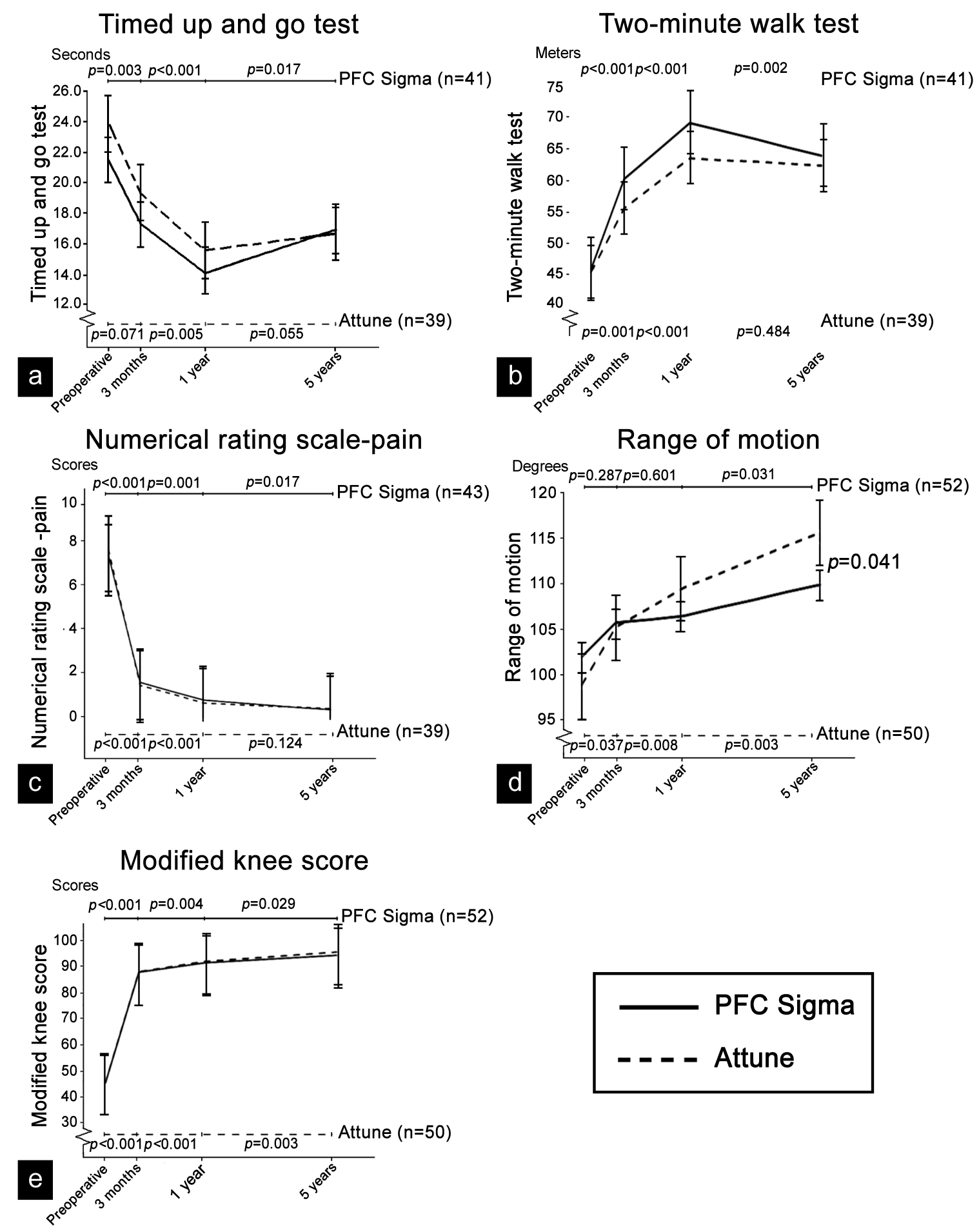

\section{Figure 1}

Mean values of each outcome measurement, and within group p-values to compare data at baseline, 3 months, 1 year, and 5 years postoperatively. (a) timed up and go test; (b) two-minute walk test; (c) numerical rating scale - pain; (d); range of motion; and, (e) modified knee score 